\title{
THE WHITEHEAD GROUP OF POLY-(FINITE OR CYCLIC) GROUPS
}

\author{
F. T. FARRELL AND W. C. HSIANG
}

\section{Introduction}

In this paper, we consider the class $\mathscr{C}$ of poly-(finite or cyclic) groups: $\Gamma \in \mathscr{C}$ if it has normal series

$$
\Gamma=\Gamma_{1} \supseteq \Gamma_{2} \supseteq \ldots \supseteq \Gamma_{n}=1
$$

such that each factor group $\Gamma_{i} / \Gamma_{i+1}$ is either a finite group or an infinite cyclic group. If all the factor groups in $(0.1)$ are $\infty$-cyclic, then $\Gamma$ is a poly- $\mathbb{Z}$ group; a group is virtually poly- $\mathbb{Z}$ (poly- $\mathbb{Z}$ by finite) if it contains a poly- $\mathbb{Z}$ subgroup of finite index. It is well known (cf. [21]) that $\mathscr{C}$ is the same as the class of virtually poly- $\mathbb{Z}$ groups. We now state the main result of this paper.

THEOREM 3.2. Let $\Gamma$ be a torsion-free, poly-(finite or cyclic) group; then Wh $\Gamma=0$ and $\tilde{K}_{0}(\mathbb{Z} \Gamma)=0$.

This extends our earlier result [14; Theorem 3.1] where we showed that Wh $\Gamma=0$ if $\Gamma$ is a Bieberbach group, that is, if $\Gamma$ is torsion-free and contains a finitely generated abelian subgroup of finite index. To prove Theorem 3.2, we make frequent use of another class of groups-crystallographic groups. A group $\hat{\Gamma}$ is crystallographic if it is a discrete cocompact subgroup of $E(n)$-the group of rigid motions of Euclidean $n$-space. The class of torsion-free crystallographic groups is identical to the class of Bieberbach groups. The intersection of a crystallographic group $\hat{\Gamma}$ with the translation subgroup of $E(n)$ is the maximal abelian subgroup of $\hat{\Gamma}$ with finite index; we denote this subgroup by $A$. It is a finitely generated normal subgroup of $\hat{\Gamma}$ and the finite factor group $\hat{\Gamma} / A-$ called the holonomy group of $\hat{\Gamma}-$ will be denoted by $G$.

The proof of Theorem 3.2 follows the same general outline of our earlier result [14; Theorem 3.1] but with some new ingredients added and some old ones modified. We briefly highlight the changes. In $\$ 1$, we use recent work of Auslander and Johnson [3] to construct a fibering apparatus $\mathscr{A}=(\hat{\Gamma}, \phi, f)$ for a torsion-free, poly-(finite or cyclic) group $\Gamma ; \mathscr{A}$ consists of a free properly discontinuous action of $\Gamma$ on $\mathbb{R}^{n}$ with compact orbit space $\mathbb{R}^{n} / \Gamma$, a non-trivial crystallographic group $\hat{\Gamma}$, an epimorphism $\phi: \Gamma \rightarrow \hat{\Gamma}$ and a $\phi$-equivariant fibration $f: \mathbb{R}^{n} \rightarrow \mathbb{R}^{m}$ with fiber diffeomorphic to $\mathbb{R}^{n-m}$.

In $\$ 2$, we define the notion of an $(\mathscr{A}, \varepsilon, h)$-cobordism; roughly speaking, this is an $h$-cobordisı. over $\mathbb{R}^{n} / \Gamma$ supporting deformation retractions generating a family of

Received 16 May, 1980; revised 1 September, 1980.

The first named author was partially supported by NSF grant Number MCS-7923654.

The second named author was partially supported by NSF grant Number GP-39324XI. 
paths in $\mathbb{R}^{n}$ each of which projects under $f$ to a path of arc length less than $\varepsilon$ in $\mathbb{R}^{m}$. We also note that the expanding immersion theorem of Epstein and Shub [9] has a meaning not only for Bieberbach groups but also for crystallographic groups.

Finally, we replace Ferry's metric vanishing theorem (cf. [14; Theorem 2.2]) by Theorem 2.3 which is valid for any admissible $(\mathscr{A}, \varepsilon, h)$-cobordism with $\varepsilon$ sufficiently small-how small depends only on $\hat{\Gamma}$. (An $(\mathscr{A}, \varepsilon, h)$-cobordism is admissible provided that $\mathrm{Wh}\left(\phi^{-1}(F)\right)=0$ and $\tilde{K}_{0}\left(\mathbb{Z} \phi^{-1}(F)\right)=0$ for every finite subgroup $F$ of $\hat{\Gamma}$.) This theorem is proven in $\S 4$; it is derived from Quinn's thin $h$-cobordism theorem [18; p. 284].

We wish to thank Bob Griess, Karl Gruenberg, Frank Raymond and Frank Quinn for helpful conversations during the preparation of this paper. We are also indebted to Terry Wall for many very helpful comments about an earlier version of this paper; for example, he showed us how to use stratification theory to clarify greatly our original proof of Theorem 2.1.

\section{Fibering apparatus}

Let $\Gamma$ be a torsion-free virtually poly- $\mathbb{Z}$ group. A fibering apparatus $\mathscr{A}=(\widehat{\Gamma}, \phi, f)$ for $\Gamma$ consists of a crystallographic group $\hat{\Gamma} \subseteq E(m)$ where $m>0$, a group epimorphism $\phi: \Gamma \rightarrow \hat{\Gamma}$, a properly discontinuous (and hence free) action of $\Gamma$ on $\mathbb{R}^{n}$ with compact orbit space and a $\phi$-equivariant fiber bundle map $f: \mathbb{R}^{n} \rightarrow \mathbb{R}^{m}$ with fiber diffeomorphic to $\mathbb{R}^{n-m}$.

THEOREM 1.1. If $\Gamma$ is a torsion-free, virtually poly- $\mathbb{Z}$ group, then $\Gamma$ has a fibering apparatus.

We wish to thank Frank Raymond for showing us how to deduce Theorem 1.1 from the techniques in $[6,7,19]$. We shall give here an alternative line of reasoning based on the work of Auslander and Johnson [3]. We first prove a special case of this result.

LemMA 1.2. If $\Gamma$ is finitely generated, torsion-free and virtually nilpotent, then $\Gamma$ has a fibering apparatus.

Proof. Let $N$ denote a normal nilpotent subgroup with finite index in $\Gamma$. By Malcev's work (cf. [21; p. 231]), there exists a simply connected (connected) nilpotent Lie group $L$ which contains $N$ as a discrete cocompact subgroup. Also, we can form the "pushout"

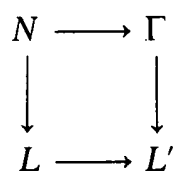

and write $L^{\prime}$ as the semi-direct product $L^{\prime}=L \rtimes F$, where $F=\Gamma / N=L^{\prime} / L$. Let $\mathbb{R}^{n}=L$ and the action of $\Gamma$ on $\mathbb{R}^{n}$ be determined by the semi-direct product structure $L^{\prime}=L \rtimes F$. (Note that $F$ embecis in $L^{\prime}$ as a maximal compact subgroup. Under this embedding, the left coset space $L^{\prime} / F$ can be canonically ides tified with $L$ and the natural action of $\Gamma$ on $L^{\prime} / F$ induced by left multiplication agrees with the one mentioned above.) 
Note that the commutator subgroup $[L, L]$ is a closed characteristic subgroup of $L$; let $\mathbb{R}^{m}$ be the factor abelian group $L /[L, L]$ and let $f$ be the canonical map $L \rightarrow L /[L, L]$, which is a fiber bundle projection with fiber $[L, L]$ diffeomorphic to $\mathbb{R}^{n-m}$. Recall next that the image of $N$ in $L /[L, L]$ under $f$ (denoted by $A$ ) is a discrete cocompact subgroup; see $[2 ;$ p. 231] or $[1 ;$ p. 4].

The action of $F$ on $L$ induces an action of $F$ on $\mathbb{P}^{m}=L /[L, L]$. Let $F_{0}$ be the kernel of this representation; then $F / F_{0}=G$ acts effectively on $\mathbb{R}^{m}$ and $f$ extends to a group homomorphism $\hat{f}: L \rtimes F \rightarrow \mathbb{R}^{m} \rtimes G$. Let $\hat{\Gamma}$ be the image of $\Gamma$ under $\hat{f}$; then $\hat{\Gamma}$ is a discrete cocompact subgroup of $\mathbb{R}^{m} \rtimes G$; hence $\hat{\Gamma}$ is a crystallographic group since $\mathbb{R}^{m} \rtimes G$ embeds in $\mathbb{R}^{m} \triangleleft O(m)=E(m)$ as a closed cocompact subgroup.

Proof of Theorem 1.1. Because of Lemma 1.2, we may assume that $\Gamma$ does not contain a nilpotent subgroup with finite index. By a result of Auslander and Johnson [3], $\Gamma$ contains a normal subgroup $G^{*}$ with finite factor group $F$ such that $\Gamma$ is a discrete cocompact subgroup of $D\left(G^{*}\right) \triangleleft F$, where $D\left(G^{*}\right)$ is a connected solvable Lie group and $D\left(G^{*}\right) \cap \Gamma=G^{*}$. Furthermore, $D\left(G^{*}\right)=R\left(G^{*}\right) \rtimes K$ where $K$ is a compact abelian Lie group and $R\left(G^{*}\right)$ is a closed connected, simply connected, solvable subgroup. Let $N$ be the nil-radical of $D\left(G^{*}\right)$. Recall that $N$ is the maximal connected nilpotent normal Lie subgroup of $D\left(G^{*}\right)$; consequently, $N$ is a closed subgroup of $D\left(G^{*}\right)$. Since $\left[D\left(G^{*}\right), D\left(G^{*}\right)\right] \subset N$, it follows that $D\left(G^{*}\right) / N$ is an abelian Lie group; let $g: D\left(G^{*}\right) \rightarrow D\left(G^{*}\right) / N$ be the canonical homomorphism. As a consequence of Mostow's structure theorem (cf. [2; p. 249]), $g\left(G^{*}\right)$ is a discrete cocompact subgroup of $D\left(G^{*}\right) / N$. Write $D\left(G^{*}\right) / N$ as $T^{s} \times \mathbb{R}^{m}$ where $T^{s}$ is the maximal compact subgroup and let $\bar{g}: D\left(G^{*}\right) \rightarrow \mathbb{R}^{m}$ be the composite

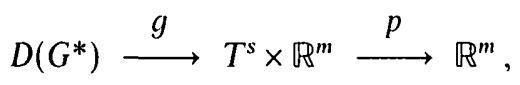

where $p$ is the canonical homomorphism, so that $\operatorname{ker} p=T^{s}$. Note that $m>0$, since otherwise $g\left(G^{*}\right)$ would be finite, contradicting the fact that $\Gamma$ does not contain a nilpotent subgroup with finite index.

The action of $F$ on $D\left(G^{*}\right)$ induces an action on $\mathbb{R}^{m}$. Let $F_{0}$ be the kernel of this representation; then $F_{1}=F / F_{0}$ acts effectively on $\mathbb{R}^{m}$ and $\bar{g}$ extends to a group homomorphism $\hat{g}: D\left(G^{*}\right) \triangleleft F \rightarrow \mathbb{R}^{m} \triangleleft F_{1}$. Let $\hat{\Gamma}=\hat{g}(\Gamma)$; then $\hat{\Gamma}$ is a discrete cocompact subgroup of $\mathbb{R}^{m} \triangleleft F_{1}$ and hence a crystallographic group. Let $K^{*}$ be a maximal compact subgroup of $D\left(G^{*}\right)>{ }^{\prime}$ containing $K$ and $K^{\prime}=\hat{g}\left(K^{*}\right)$ which is a maximal compact subgroup of $\mathbb{R}^{m} \triangleleft F_{1}$. Then $\hat{g}$ induces a $\phi$-equivariant map $f$ between the corresponding left coset spaces,

$$
f: D\left(G^{*}\right) \triangleleft F / K^{*} \longrightarrow \mathbb{R}^{m} \triangleleft F_{1} / K^{\prime}
$$

where $\phi=\hat{g} \mid \Gamma$ and $\Gamma$ (respectively, $\hat{\Gamma}$ ) acts on $D\left(G^{*}\right) \triangleleft F / K^{*}$ (respectively, $\left.\mathbb{R}^{m} \rtimes F_{1} / K^{\prime}\right)$ by left multiplication. But $f$ is a fiber bundle projection with base space, total space and fiber diffeomorphic to $\mathbb{R}^{m}, \mathbb{B}^{n}$ and $\mathbb{R}^{n-m}$ (respectively).

\section{2. $(\propto, \varepsilon, h)$-cobordisms}

Let $\Gamma$ be a torsion-free, virtually poly- $\mathbb{Z}$ group and let $\mathscr{A}=(\hat{\Gamma}, \phi, f)$ be a fibering apparatus for $\Gamma$. Let $K$ be a closed smooth simply connected manifold such 
that $\operatorname{dim} K+n>4$ where $n=\operatorname{cd} \Gamma$. (See the proof of Theorem 3.2 for a discussion of cd $\Gamma$.) Consider a smooth $h$-cobordism $\left(W ; \partial_{0} W, \partial_{1} W\right)$ such that $\partial_{0} W=\left(\mathbb{B}^{n} / \Gamma\right) \times K$; it is an $(\mathscr{A}, \varepsilon, h)$-cobordism (where $\varepsilon>0$ is a real number) provided there exist smooth deformation retractions $h_{t}, k_{t}: W \times[0,1] \rightarrow W$ onto $\partial_{0} W, \partial_{1} W$ (respectively) such that each path in a certain associated family $\left\{\alpha_{x}, \gamma_{x} \mid x \in \bar{W}\right\}$ (in $\mathbb{R}^{m}$ ) has arc length less than $\varepsilon$ (where $\bar{W}$ denotes the universal cover of $W$ ). These paths are defined as follows. Let $\bar{h}_{t}, \bar{k}_{t}: W \times[0,1] \rightarrow \bar{W}$ be the liftings of $h_{t}, k_{t}$ (respectively) such that $\bar{h}_{0}=\bar{k}_{0}=$ id; then

$$
\alpha_{x}(t)=f p \bar{h}_{1} \bar{h}_{t}(x) \quad \text { and } \quad \gamma_{x}(t)=f p \bar{h}_{1} \bar{k}_{t}(x) \quad \text { for } \quad 0 \leqslant t \leqslant 1,
$$

where $p: \mathbb{R}^{n} \times K \rightarrow \mathbb{R}^{n}$ is the projection defined by the product structure.

A fibering apparatus $\mathscr{A}=(\hat{\Gamma}, \phi, f)$ is admissible provided, for each finite subgroup $F$ of $\hat{\Gamma}$, both $W h \phi^{-1}(F)$ and $\tilde{K}_{0}\left(\mathbb{Z} \phi^{-1}(F)\right)$ vanish. The following result can be derived from Quinn's thin $h$-cobordism theorem $[18 ;$ p. 284].

THEOREM 2.1. Let $\hat{\Gamma} \subseteq E(m)$ be a crystallographic group; then there exists a real number $\varepsilon>0$ with the following property. Let $\Gamma$ be any torsion-free, virtually poly- $\mathbb{Z}$ group with an admissible fibering apparatus $\mathscr{A}=(\hat{\Gamma}, \phi, f)$; then the Whitehead torsion (calculated in $\mathrm{Wh} \Gamma)$ of any $(\mathscr{A}, \varepsilon, h)$-cobordism vanishes.

The proof of this result is deferred until $\S 4$.

Let $\Gamma$ be a torsion-free, virtually poly- $\mathbb{Z}$ group and let $\mathscr{A}=(\hat{\Gamma}, \phi, f)$ be a fibering apparatus for $\Gamma$. Let $G$ be the holonomy group of $\hat{\Gamma}$ and $A$ the maximal abelian subgroup of $\hat{\Gamma}$ of finite index; recall that $\hat{\Gamma} / A=G$. By a slight extension of the terminology of [14], we say that a monomorphism $\psi: \hat{\Gamma} \rightarrow \hat{\Gamma}$ is s-expansive if $\psi$ induces multiplication by $s$ on $A$ (where $s$ is a positive integer) and the identity map on $G$. We say that a subgroup $\Gamma^{\prime}$ of finite index in $\Gamma$ has level $(s, \phi)$ provided $\Gamma^{\prime}=\phi^{-1}(\psi(\hat{\Gamma}))$ for some s-expansive monomorphism $\psi: \hat{\Gamma} \rightarrow \hat{\Gamma}$. We need the following immediate extension of the Epstein-Shub result [9] from Bieberbach groups to crystallographic groups. (The Bieberbach case was used in [14].) In fact, the following result was implicitly proven in [9].

THEOREM 2.2. For any positive integer $s \equiv 1 \bmod |G|$, there exists an s-expansive endomorphism $\psi$ of $\hat{\Gamma}$. Furthermore, for any s-expansive endomorphism $\psi$ of $\hat{\Gamma}$, there exists a $\psi$-equivariant diffeomorphism $g: \mathbb{R}^{m} \rightarrow \mathbb{R}^{m}$ (relative to $\hat{\Gamma} \subseteq E(m)$ ) such that $|d g(X)|=s|X|$ for each vector $X$ tangent to $\mathbb{R}^{m}$, where || is the Euclidean metric on $\mathbb{R}^{m}$.

We now apply Theorems 2.1 and 2.2 to obtain a vanishing result for the transfer map.

THEOREM 2.3. If $\mathscr{A}=(\hat{\Gamma}, \phi, f)$ is an admissible fibering apparatus for $\Gamma$, then to each element $b \in \mathrm{Wh} \Gamma$ there corresponds an integer $N(b, \mathscr{A})$ with the following property. For every subgroup $\Gamma^{\prime}$ of level $(s, \phi)$ with $s>N(b, \mathscr{A}), \omega^{*}(b)=0$ where $\omega: \Gamma^{\prime} \rightarrow \Gamma$ denotes the inclusion map and $\omega^{*}: \mathrm{Wh} \Gamma \rightarrow \mathrm{Wh} \Gamma^{\prime}$ denotes the induced transfer homomorphism. 
Proof. Represent $b$ as the torsion of an $h$-cobordism $\left(W ; \partial_{0} W, \partial_{1} W\right)$ with $\partial_{0} W=\mathbb{R}^{n} / \Gamma \times S^{5}$ where $S^{5}$ denotes the 5 dimensional sphere. (If $n>4$, then $S^{5}$ is unnecessary, that is we can assume $\partial_{0} W=\mathbb{R}^{n} / \Gamma$.) Let $h_{t}, k_{t}: W \times[0,1] \rightarrow W$ be smooth deformation retractions onto $\partial_{0} W, \partial_{1} W$ (respectively); then there exists a real number $U$ such that the arc length of each path $\alpha_{x}$ or $\gamma_{x}$ in the family $\left\{\alpha_{x}, \gamma_{x} \mid x \in \bar{W}\right\}$ is less than $U$, where $\alpha_{x}$ and $\gamma_{x}$ are defined by equations (2.1).

Let $\varepsilon>0$ be the real number (dependent on $\hat{\Gamma}$ ) posited in Theorem 2.1; then pick $N(b, \mathscr{A})$ to be any positive integer larger than $U / \varepsilon$. Let $\psi: \hat{\Gamma} \rightarrow \hat{\Gamma}$ be an $s$-expansive endomorphism such that $\psi(\hat{\Gamma})=\phi\left(\Gamma^{\prime}\right)$ and let $g: \mathbb{R}^{m} \rightarrow \mathbb{R}^{m}$ be the $\psi$-equivariant diffeomorphism posited in Theorem 2.2. Then, $\mathscr{A}^{\prime}=\left(\hat{\Gamma}, \psi^{-1}\left(\phi \mid \Gamma^{\prime}\right), g^{-1} f\right)$ is a fibering apparatus for $\Gamma^{\prime}$ and the finite sheeted covering space $W^{\prime}$ of $W$ corresponding to $\Gamma^{\prime} \subseteq \Gamma$ is an $\left(\mathscr{A}^{\prime}, \varepsilon, h\right)$-cobordism. Hence, by Theorem 2.1 , the torsion of $W^{\prime}$ vanishes in Wh $\left(\Gamma^{\prime}\right)$; but this torsion is $\omega^{*}(b)$.

We shall need the following $\bar{K}_{0}$-analogue of the above result.

Corollary 2.4. If $\mathscr{A}=(\hat{\Gamma}, \phi, f)$ is an admissible fibering apparatus for $\Gamma$, then to each element $b \in \bar{K}_{0}(\mathbb{Z} \Gamma)$ there corresponds an integer $\bar{N}(b, \mathscr{A})$ with the following property. For every subgroup $\Gamma^{\prime}$ of level $(s, \phi)$ with $s>\bar{N}(b, \mathscr{A}), \omega^{*}(b)=0$ where $\omega: \Gamma^{\prime} \rightarrow \Gamma$ denotes the inclusion map and $\omega^{*}: \tilde{K}_{0}(\mathbb{Z} \Gamma) \rightarrow \tilde{K}_{0}\left(\mathbb{Z} \Gamma^{\prime}\right)$ denotes the induced transfer homomorphism.

We deduce this from Theorem 2.3 together with Lemmas 2.5 and 2.6 from [14]. We need a slightly modified version of Lemma 2.5 of [14] which is easily verified.

Lemma 2.5'. Let $\omega: \Gamma^{\prime} \rightarrow \Gamma$ be the inclusion of a subgroup of finite index; then the diagram

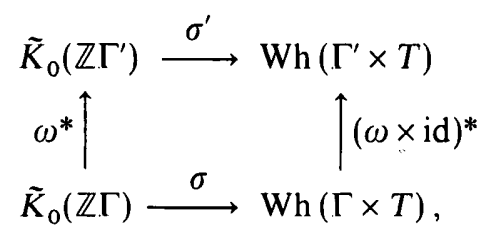

where $T$ is the $\infty$-cyclic group and $\sigma, \sigma$ are the canonical embeddings described in $[14 ;$ p. 186], commutes.

Proof of Corollary 2.'4. Set $\bar{N}(b, \mathscr{A})=N(\sigma(b), \overline{\mathscr{q}})$, where $\bar{s}=(\hat{\bar{\Gamma}} \times T$, $\phi \times$ id, $f \times$ id) is the fibering apparatus for $\Gamma \times T$ determined from $\mathscr{A}$. (Note that $T \subseteq E(1)$ is the subgroup of all translations of $\mathbb{R}$ of the form $x \mapsto x+t$ where $t$ is an integer, and the actions of $\Gamma \times T$ and $\hat{\Gamma} \times T$ on $\mathbb{R}^{n} \times \mathbb{R}$ and $\mathbb{R}^{m} \times \mathbb{R}$, respectively, are canonically induced.) By Lemma 2.6 of [14], it suffices to show that (id $\times g)^{*} \sigma^{\prime} \omega^{*}(b)=0$, where $g: T \rightarrow T$ is multiplication by $s$. Note that $g$ factors as the composite $\bar{\omega} \bar{g}$ where $\bar{\omega}: s T \rightarrow T$ is the inclusion map and $\bar{g}: T \rightarrow s T$ is an isomorphism. (We define $s T$ to be the subgroup of $T$ consisting of all elements divisible by $s$.) Using Lemma 2.5', we have

$$
(\mathrm{id} \times g)^{*} \sigma^{\prime} \omega^{*}(b)=(\mathrm{id} \times \bar{g})^{*}(\omega \times \bar{\omega})^{*} \sigma(b) ;
$$

but by Theorem $2.4,(\omega \times \bar{\omega})^{*} \sigma(b)=0$ since $\Gamma^{\prime} \times s T \subseteq \Gamma \times T$ has level $(s, \phi \times$ id $)$. 


\section{The main result}

Let $\hat{\Gamma}$ be a crystallographic group, $A$ its maximal abelian subgroup of finite index and $G=\hat{\Gamma} / A$ its holonomy group. For any positive integer $s$, define $\hat{\Gamma}_{s}=\hat{\Gamma} / s A$ and $A_{s}=A / s A$ where $s A$ is the subgroup of $A$ consisting of all elements divisible by $s ; \hat{\Gamma}_{s}$ is an extension of $A_{s}$ by $G$ which is a semidirect product if $(s,|G|)=1$. Let $T$ denote the infinite cyclic group.

THEOREM 3.1. Let $\hat{\Gamma}$ be a crystallographic group with holonomy group $G$; then

(i) $\hat{\Gamma}=\Pi>T$, or

(ii) $\hat{\Gamma}=B * C$ where $D$ has index 2 in both $B$ and $C$, or

(iii) there is an infinite sequence of positive integers $s$ with $s \equiv 1 \bmod |G|$ such that any hyperelementary subgroup of $\hat{\Gamma}_{s}$ which projects onto $G$ (via the canonical map) projects isomorphically to $G$.

Note that this result extends Theorem 1.1 of [14] from the class of Bieberbach groups to that of crystallographic groups. The proof is the same as before with one modification; namely, in the penultimate paragraph on page 184 of [14] we cannot use Lemma 4.1 of [13] since this result is only true for Bieberbach groups. Instead, argue as follows in the case when $p \backslash s$ (referring to the line of reasoning and notation used in $[14 ;$ p. 184, Proof of Theorem 1.1]). Note that the hyperelementary group $S$ is a semidirect product; namely, $S=T_{k} \triangleleft \triangleleft P$ where $T_{k}$ is cyclic of order $k$ and $P$ is a $p$-group. Since $p \backslash s, P \subseteq A_{s}$ and $T_{k}$ projects onto $G$ (via the canonical map); therefore $A_{s} \cap T_{k} \subseteq\left(A_{s}\right)^{G}$. If $\left|A_{s} \cap T_{k}\right|>1$, then $\hat{\Gamma}=\Pi>\triangleleft T$ by [14; Lemmas 1.2 and 1.4]. If $\left|A_{s} \cap T_{k}\right|=1$, then $P$ is a normal subgroup of $S$ and in fact $S=P \times T_{k}$. Hence $P=S \cap A_{s} \subseteq\left(A_{s}\right)^{G}$ and if $\left|S \cap A_{s}\right|>1$, then $\hat{\Gamma}=\Pi \rtimes T$ by [14; Lemmas 1.2 and 1.4]. But, if $\left|S \cap A_{s}\right|=1$, then (iii) is satisfied. This completes the modification to the proof of [14; Theorem 1.1] necessary to prove Theorem 3.1.

We wish to point out that Dan Farkas had proven Lemma 1.4 of [14] many years before us (cf. [10; p. 432]).

We now formulate the major result of this paper.

THEOREM 3.2. Let $\Gamma$ be a torsion-free virtually poly- $\mathbb{Z}$ group. Then $\mathrm{Wh} \Gamma=0$ and $\tilde{K}_{0}(\mathbb{Z} \Gamma)=0$.

Proof. Recall that the cohomological dimension of $\Gamma$, denoted by $\operatorname{cd}(\Gamma)$, is the largest integer $n$ such that $H^{n}\left(\Gamma, \mathbb{Z}_{2}\right) \neq 0 ; \operatorname{cd}(\Gamma)$ is identical to the dimension of any closed aspherical manifold with fundamental group $\Gamma$. Define the holonomy number of $\Gamma$, denoted by $h(\Gamma)$, to be the minimum order of the holonomy group of a crystallographic group $\hat{\Gamma}$ that can occur in a fibering apparatus $(\hat{\Gamma}, \phi, f)$ for $\Gamma$. We proceed in our proof by induction first on cd $(\Gamma)$ and next on $h(\Gamma)$; that is, we assume that $\mathrm{Wh} \Pi=0$ and $\tilde{K}_{0}(\mathbb{Z} \Pi)=0$ for all torsion-free virtually poly- $\mathbb{Z}$ groups $\Pi$ where either $\operatorname{cd}(\Pi)<\operatorname{cd}(\Gamma)=n$, or both $\operatorname{cd}(\Pi)=n$ and $h(\Pi)<h(\Gamma)=i$.

Let $\mathscr{A}=(\hat{\Gamma}, \phi, f)$ be a fibering apparatus for $\Gamma$ such that $|G|=h(\Gamma)$, where $G$ is the holonomy group of $\hat{\Gamma}$. Start by considering $\mathrm{Wh} \Gamma$; Theorem 3.1 shows there are 
three possibilities, (i), (ii) and (iii), for the structure of $\hat{\Gamma}$. In (i), $\hat{\Gamma}=\bar{\Gamma} \rtimes T$ and hence $\Gamma=\Pi \rtimes T$ where $\Pi=\phi^{-1}(\bar{\Gamma})$. Since $\mathbb{Z} \Pi$ is right regular, we have by [12] the exact sequence

$$
\text { Wh } \Pi \longrightarrow \mathrm{Wh} \Gamma \longrightarrow \tilde{K}_{0}(\mathbb{Z} \Pi)
$$

Since cd $(\Pi)<n$, both $W h \Pi$ and $\tilde{K}_{0}(\mathbb{Z} \Pi)$ vanish; hence Wh $\Gamma=0$. In the case of possibility (ii), $\hat{\Gamma}=B \underset{D}{*} C$ where $D$ has index 2 in both $B$ and $C$; hence $\Gamma=B^{\prime}{ }_{D^{\prime}}^{*} C^{\prime}$ where $B^{\prime}=\phi^{-1}(B), C^{\prime}=\phi^{-1}(C)$ and $D^{\prime}=\phi^{-1}(D)$. We have by [20] an exact sequence

$$
\mathrm{Wh}\left(B^{\prime}\right) \oplus \mathrm{Wh}\left(C^{\prime}\right) \longrightarrow \mathrm{Wh} \Gamma \longrightarrow \tilde{K}_{0}\left(\mathbb{Z} D^{\prime}\right)
$$

which yields $\mathrm{Wh} \Gamma=0$ by our induction hypothesis since $\operatorname{cd}\left(B^{\prime}\right)=\operatorname{cd}\left(C^{\prime}\right)=\operatorname{cd}\left(D^{\prime}\right)=n-1$. If possibility (iii) applies, let $b \in \mathrm{Wh} \Gamma$ be arbitrary and $s$ be one of the integers given by Theorem 3.1 subject to the added constraint that $s>N(b, \mathscr{A})$, where $N(b, \mathscr{A})$ is the integer posited in Theorem 2.3. (Note that $\mathscr{A}$ is an admissible fibering apparatus for $\Gamma$; this is a consequence of our induction hypothesis since $\operatorname{cd}\left(\phi^{-1}(F)\right)<\operatorname{cd}(\Gamma)$ for each finite subgroup $F$ of $\hat{\Gamma}$.) Now apply Frobenius induction to Wh $\Gamma$ relative to the factor group $\hat{\Gamma}_{s}$. (Recall that $\hat{\Gamma}_{s}$ is a factor group of $\hat{\Gamma}$ and hence of $\Gamma$ via $\phi$.) Let $p: \Gamma \rightarrow \hat{\Gamma}_{s}$ denote the composite of $\phi$ and the canonical homomorphism $q: \hat{\Gamma} \rightarrow \hat{\Gamma}_{s}$. As $S$ varies over the subgroups of $\hat{\Gamma}_{s}$, Wh $\left(p^{-1} S\right)$ is a Frobenius module over Swan's Frobenius functor $G_{0}(S)$ (cf. [15]). Hence, it suffices to show that $b$ vanishes under the transfer maps associated to the hyperelementary subgroups $E$ of $\hat{\Gamma}_{s}$. If $E$ projects (via the canonical map) to a proper subgroup of $G$, then the holonomy group of the crystallographic group $\bar{\Gamma}=q^{-1}(E)$ has order less than $|G|$. Let $\Gamma^{\prime}=p^{-1}(E)$; then $\mathscr{A}^{\prime}=\left(\bar{\Gamma}, \phi \mid \Gamma^{\prime}, f\right)$ is a fibering apparatus for $\Gamma^{\prime}$, and hence $h\left(\Gamma^{\prime}\right)<|G|=h(\Gamma)$. Therefore, Wh $\Gamma^{\prime}=0$ by the induction hypothesis; consequently, $\omega^{*}(b)=0$ where $\omega: \Gamma^{\prime} \rightarrow \Gamma$ is the inclusion map. Otherwise, Theorem 3.1 says that $E$ projects isomorphically onto $G$; but all such subgroups of $\hat{\Gamma}_{s}$ are conjugate since $H^{1}\left(G ; A_{s}\right)=0$. (Recall that $A$ denotes the maximal abelian subgroup of finite index in $\hat{\Gamma}$.) Hence, it suffices to consider one of them; for example, let $E=q \Psi(\hat{\Gamma})$ where $\Psi: \hat{\Gamma} \rightarrow \hat{\Gamma}$ is $s$-expansive. (The existence of $\Psi$ is a consequence of Theorem 2.2.) Let $\Gamma^{\prime}=p^{-1}(E)$; then $\Gamma^{\prime}$ has level $(s, \phi)$ since $\Gamma^{\prime}=\phi^{-1}(\Psi(\hat{\Gamma}))$. Therefore, we can apply Theorem 2.3 to obtain $\omega^{*}(b)=0$ where $\omega: \Gamma^{\prime} \rightarrow \Gamma$ denotes the inclusion map. Hence $b$ vanishes under all the appropriate transfer maps; this implies that $b=0$ and completes the inductive argument to show that $\mathrm{Wh} \Gamma=0$.

To show $\tilde{K}_{0}(\mathbb{Z} \Gamma)$ vanishes, we proceed similarly. If case (i) of Theorem 3.1 applies, then (as above) $\Gamma=\Pi \rtimes T$. Hence, we have by [12] an epimorphism $\tilde{K}_{0}(\mathbb{Z} \Pi) \rightarrow \tilde{K}_{0}(\mathbb{Z} \Gamma)$; therefore, $\tilde{K}_{0}(\mathbb{Z} \Gamma)=0$. If case (ii) applies, then (as above) $\Gamma=B^{\prime}{ }_{D^{\prime}}^{*} C^{\prime}$ where $D^{\prime}$ has index 2 in both $B^{\prime}$ and $C^{\prime}$. By $[4], \widetilde{K}_{0}(\mathbb{Z} \Gamma)$ is isomorphic to a subgroup of $\mathrm{Wh}(\Gamma \times T)$; hence, it suffices to show that $\mathrm{Wh}(\Gamma \times T)=0$. But this is done exactly as in the last paragraph on page 187 of [14]. Finally, consider the situation where case (iii) of Theorem 3.1 applies. Then, proceed exactly as in the similar situation for Wh $\Gamma$ considered above; that is, apply Frobenius induction to $\tilde{K}_{0}(\mathbb{Z} \Gamma)$ using Corollary 2.4 in place of Theorem 2.3 to see that $\tilde{K}_{0}(\mathbb{Z} \Gamma)=0$. This completes the proof of Theorem 3.2. 


\section{Proof of Theorem 2.1}

We first formulate a variant of Quinn's thin $h$-cobordism theorem; it will be used to prove Theorem 2.1. Let $X$ be a compact Riemannian manifold and $C$ be a codimension- 0 submanifold of $X$ such that $\partial X \cap C=\varnothing$. Let $N^{n}$ be a compact smooth manifold and $E^{n}$ be a codimension- 0 submanifold (with corners) of $N^{n}$ such that $\partial_{0} E=E \cap \partial N$ is a codimension-0 submanifold of $\partial E$; let $\partial_{1} E=\partial E-\partial_{0} E$. (Note that $E$ has a corner at $\partial_{0} E \cap \partial_{1} E$.) Let $p: E \rightarrow X$ be a fiber bundle projection with fiber $S$ such that $\mathrm{Wh}\left(\pi_{1} S \times \mathbb{Z}^{i}\right)=0$ for all $i \geqslant 0$ where $\mathbb{Z}^{i}$ denotes the free abelian group of rank $i$. Let $W^{n+1}$ be a compact smooth manifold containing $N^{n}$ as a codimension- 0 submanifold of $\partial W$; denote $N^{n}$ by $\partial_{-} W$ and the closure of $\partial W^{n+1}-N^{n}$ by $\partial_{+} W$. Let $U$ be an open subset of $W$ such that $E \subset \partial U \subset \partial W$ and $h_{t}, k_{t}: U \times[0,1] \rightarrow W$ be homotopies such that
(1) $h_{0}=k_{0}=$ identity;
(2) image $\left(h_{1}\right) \subset \partial_{-} W$ and image $\left(k_{1}\right) \subset \partial_{+} W$;
(3) if $x \in \partial_{-} W \cap U$, then $h_{t}(x) \equiv x$;
(4) likewise, if $x \in \partial_{+} W \cap U$, then $k_{t}(x) \equiv x$;
(5) $h_{1}^{-1}(E)$ is compact.

If $\delta>0$, we say that $\left(W^{n+1}, N^{n}\right)$ is a $(\delta, h)$-cobordism over a compact set $K \subset X$ (relative to $U, h_{t}, k_{t}$ ) provided that

(1) $d(K, \partial X)>\delta$ where $d($, $)$ is the metric on $X$;

(2) for each $x \in h_{1}^{-1}\left(p^{-1}(K)\right)$ define $\alpha_{x}(t)=h_{1}\left(h_{t}(x)\right)$ and $\gamma_{x}(t)=h_{1}\left(k_{t}(x)\right)$ (for $0 \leqslant t \leqslant 1$ ); then $\alpha_{x}, \gamma_{x}$ are curves in $E$ and the diameters (in $X$ ) of the images of the composite curves $p \alpha_{x}, p \gamma_{x}$ are less than $\delta$.

For $\varepsilon>0$ and $B$ a subset of a metric space $Y$, define $B^{\varepsilon}$ by

$$
B^{\varepsilon}=\{y \mid y \in Y, d(y, B)<\varepsilon\},
$$

where $d($,$) denotes the metric on Y$. We say that an embedding $F: p^{-1}\left(K^{\delta}\right) \times[0,1] \rightarrow W$ is a $\delta$-product structure over $K$ relative to $h_{1}$ provided that

(1) $F(x, 0) \equiv x$;

(2) $h_{1}^{-1}\left(p^{-1}(K)\right) \subset$ image $(F)$;

(3) for each $x \in p^{-1}(K), h_{1}(F(x, t)) \in E$ and the diameter (in $X$ ) of $\left\{p h_{1}(F(x, t)) \mid 0 \leqslant t \leqslant 1\right\}$ is less than $\delta$.

TheOREM 4.1. Given $\varepsilon>0$ with $4 \varepsilon<d(C, \partial X)$, there exists a $\delta>0$ depending only on $\varepsilon, X$ and $C$ (in particular, $\delta$ is independent of $p$ ) such that any $(\delta, h)$-cobordism $\left(W^{n+1}, N^{n}\right)$ over the closure of $C^{2 \varepsilon}$ (relative to $\left.U, h_{t}, k_{t}\right)$ has an $\varepsilon$-product structure over $C$ relative to $h_{1}$ (provided that $n>4$ ). 
Proof. The submanifold $C$ has a handlebody decomposition with a finite number of handles $H_{1}, H_{2}, \ldots, H_{m}$ attached in the order of their numerical index. Since the bundle $p: E \rightarrow X$ is trivial over each handle $H_{i}$, we can use Quinn's thin $h$-cobordism theorem [18; Theorem 2.7] to put first a product structure over $H_{1}$ and then extend this structure inductively over a handle at a time so that after $m$ steps we obtain the desired product structure over $C$. This is an outline of the proof; the details are left as an exercise for the reader.

We next formulate the relative version of Theorem 4.1 ; it will be the main ingredient in the proof of Theorem 2.1. Suppose we have a product structure already given in an open neighbourhood $V$ of $\partial N$ in $N$; that is, we are given an embedding $F: V \times[0,1] \rightarrow W$ such that

$$
\begin{aligned}
& \text { (1) } F \mid V \times 0=\text { identity; } \\
& \text { (2) }(F(\partial N \times[0,1]) \cup F(V \times 1)) \subset \partial_{+} W .
\end{aligned}
$$

We wish to extend this structure in a metrically controlled way over $C$. To do this, we slightly modify the notion of $(\delta, h)$-cobordism over $C$. Let $\partial_{-1} W$ denote $N=\partial_{-} W$, let $\partial_{0} W=F(\partial N \times[0,1])$ and let $\partial_{1} W$ be the closure of $\partial_{+} W-\partial_{0} W$. Let $U$ be an open subset of $W$ such that

$$
\left(E \cup F\left(\partial_{0} E \times[0,1]\right) \cup F(E \cap V \times 1)\right) \subset \partial U \subset \partial W
$$

and $h_{t}, k_{t}: U \times[0,1] \rightarrow W$ be homotopies satisfying both property (4.1) in which $\partial_{-} W, \partial_{+} W$ are replaced by $\partial_{-1} W, \partial_{1} W$, respectively, and the following property:

$$
\begin{aligned}
& \text { if } F(x, t) \in U \text { and } 0 \leqslant s \leqslant 1 \text {, then } h_{s}(F(x, t))=F(x,(1-s) t) \text { and } \\
& k_{s}(F(x, t))=F(x, t+s(1-t)) .
\end{aligned}
$$

We say that $\left(W^{n+1}, N^{n}\right)$ is a $(\delta, h)$-cobordism over a compact set $K \subset X$ with product structure $F$ near $\partial N$ (relative to $U, h_{t}, k_{t}$ ) provided property (4.2) is satisfied and $U, h_{t}, k_{t}$ satisfy $(4.1),(4.6)$ and (4.7).

Corollary 4.2. Given $\varepsilon>0$ with $4 \varepsilon<d(C, \partial X)$ and $n>4$, there exists a $\delta>0$ depending only on $\varepsilon, X$ and $C$ such that any $(\delta, h)$-cobordism $\left(W^{n+1}, N^{n}\right)$ over the closure of $C^{2 k}$ with a product structure $F$ near $\partial N$ (relative to $U, h_{t}, k_{t}$ ) has an $\varepsilon$-product structure $\tilde{F}$ over $C$ relative to $h_{1}$ such that $\tilde{F}(x, t)=F(x, t)$ provided $x \in \partial_{0} E, p(x) \in C^{\varepsilon}$ and $0 \leqslant t \leqslant 1$.

Proof. First, we construct a cobordism $\left(W^{\prime}, N^{\prime}\right)$ (using $\left.(W, N)\right)$ which satisfies the hypotheses of Theorem 4.1. Let $N^{\prime}=N \cup \partial_{0} W, W^{\prime}=W$ and define $\partial_{+} W^{\prime}$, $\partial_{-} W^{\prime}$ as before relative to $N^{\prime}$. Let $E^{\prime}=E \cup F\left(\partial_{0} E \times[0,1]\right)$ and define $p^{\prime}: E^{\prime} \rightarrow X$ by

$$
\begin{array}{ll}
p^{\prime}(x)=p(x) & \text { if } x \in E, \quad \text { and } \\
p^{\prime}(F(x, t))=p(x) & \text { if } x \in \partial_{0} E \quad(0 \leqslant t \leqslant 1) .
\end{array}
$$


Note that $p^{\prime}: E^{\prime} \rightarrow X$ is a fiber bundle and its fiber is homeomorphic to the fiber of $p: E \rightarrow X$; hence, $p^{\prime}$ satisfies the hypotheses of Theorem 4.1. Let $U^{\prime}=U$; then $E^{\prime} \subset \partial U^{\prime} \subset \partial W^{\prime}$ because of (4.6); also, let $k_{t}^{\prime}=k_{t}$. To construct $h_{t}^{\prime}$, put a 'nice' collar on $N^{n}$. Namely, let $G: \partial N \times[0,1] \rightarrow N$ be an embedding such that

(1) $G \mid \partial N \times 0=$ identity;

(2) image $(G) \subset V$;

(3) if $x \in \partial_{0} E$ and $0 \leqslant t \leqslant 1$, then $G(x, t) \in E$ and $p(G(x, t))=p(G(x, 0))$.

Using $G$ and $F$, we obtain an embedding $H: \partial N \times[0,1] \times[0,1] \rightarrow W$ defined by $H(x, s, t)=F(G(x, s), t)$. Note that, by $(4.7)$, the homotopy $h_{t}$ inside image $(H)$ follows the "vertical" lines $t \mapsto H(x, s, t)$, that is, it follows the images under $H(x,$, (for $x \in \partial N$ ) of the dashed vertical lines in $[0,1] \times[0,1]$ which are illustrated in the left half of Figure 1.
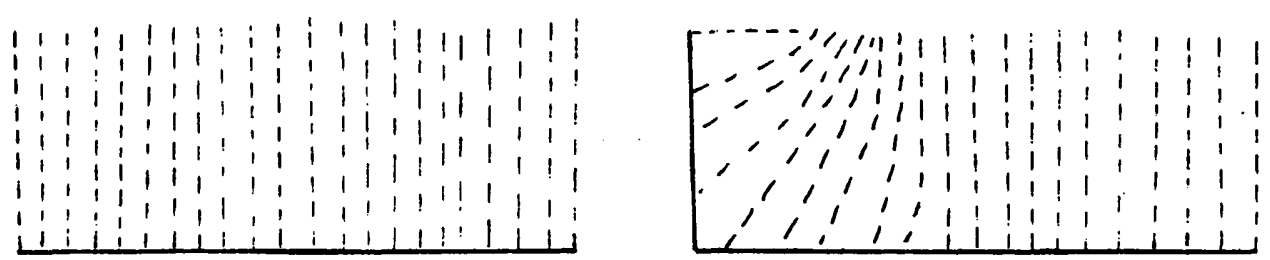

Figure 1

If we instead follow the images under $H(x,$,$) of the bent lines in [0,1] \times[0,1]$ illustrated in the right half of Figure 1 by dashed lines, we obtain a new homotopy $h_{t}^{\prime}$ such that property (4.1) is satisfied when $h_{t}, k_{t}, W, U, E$ are replaced by $h_{t}^{\prime}, k_{t}^{\prime}, W^{\prime}$, $U^{\prime}, E^{\prime}$, respectively. In addition, we have the following properties:

(1) $p^{\prime}\left(h_{1}^{\prime}(x)\right)=p\left(h_{1}(x)\right)$ if $x \in h_{1}^{-1}(E)$

(2) $\left(h_{1}^{\prime}\right)^{-1}(E)=h_{1}^{-1}(E)$;

(3) the composite curves $p^{\prime} \gamma_{x}^{\prime}$ and $p^{\prime} \alpha_{x}^{\prime}$ are the same as $p \gamma_{x}$ and $p \alpha_{x}$ (respectively) provided $x \in\left(h_{1}^{\prime}\right)^{-1}\left(p^{-1}(K)\right)$ where $K$ denotes the closure of $C^{2 \varepsilon}, \alpha_{x}^{\prime}(t)=h_{1}^{\prime}\left(h_{t}^{\prime}(x)\right)$ and $\gamma_{x}^{\prime}(t)=h_{1}^{\prime}\left(k_{t}^{\prime}(x)\right)$.

In particular, $\left(W^{\prime}, N^{\prime}\right)$ is a $(\delta, h)$-cobordism over $C^{2 \varepsilon}$ (relative to $\left.U^{\prime}, h_{t}^{\prime}, k_{t}^{\prime}\right)$. Hence, let the number $\delta$ in Corollary 4.2 be the same as the number $\delta$ posited in Theorem 4.1. Then, the hypotheses of Theorem 4.1 are satisfied by $\left(W^{\prime}, N^{\prime}\right)$ and we obtain an $\varepsilon$-product structure $F^{\prime}$ for $\left(W^{\prime}, N^{\prime}\right)$ over $C$ relative to $h_{1}^{\prime}$.

It is easy to construct a homeomorphism $L: \partial_{-1} W \times[0,1] \rightarrow \partial_{-} W^{\prime} \times[0,1]$ such that

(1) $L(x, 0)=(x, 0)$ if $x \in N=\partial_{-1} W$
(2) $L(x, t)=(F(x, t), 0)$ if $x \in \partial N(0 \leqslant t \leqslant 1)$;

(3) the family of vertical lines $t \mapsto(x, t)$ in $\partial_{-} W \times[0,1]$ (where $x \in\left[\partial_{0} W \cup\right.$ image $\left.(G)\right]$ ) is transformed by the composite map $F L^{-1}$ into the same family of curves in $W$ as are obtained by applying the maps $H(x,$,$) (for x \in \partial N)$ to the bent lines in $[0,1] \times[0,1]$ illustrated in the right half of Figure 1 by dashed lines. 
We now define the product structure $\tilde{F}$, posited in Corollary 4.2 , by the composition $\tilde{F}(x, t)=F^{\prime}(L(x, t))$ where $x \in p^{-1}\left(C^{\varepsilon}\right)$ and $t \in[0,1]$. Using (4.10), (4.11), we see that $\tilde{F}$ is an $\varepsilon$-product structure over $C$ relative to $h_{1}$. This completes the proof of Corollary 4.2 .

Next, we recall some elementary facts about a smooth action of a finite group $G$ on a closed manifold $M$. (Some general references are [5] and [8].) lior $x, y \in M$, we say that $x, y$ are of the same orbit type if their isotropy subgroups $G_{x}, G_{y}$ are conjugate. If $(H)$ is the conjugacy class of the subgroup $H$ of $G$, then $M_{(H)}$ denotes the submanifold (generally not closed) of $M$ consisting of the points whose isotropy subgroup is in $(H)$; that is

$$
M_{(H)}=\left\{x \mid x \in M, G_{x} \in(H)\right\}
$$

$M_{(H)}$ is an invariant subset of $M$ under $G$. Partition $M$ into the $G$-orbits of the connected components of the sets $M_{(H)}$ these are the strata $X$ of a stratification $\mathscr{X}$ of $M$.

Now let us consider the action of $\hat{\Gamma}$ on $\mathbb{B}^{m}$. The decomposition $1 \rightarrow^{-} A \rightarrow \hat{\Gamma} \rightarrow G \rightarrow 1$ factors the action into two steps. The subgroup $A$ acts on $\mathbb{R}^{m}$ freely and the orbit space, denoted by $M^{m}$, is a flat torus. Also, the finite group $G$ acts on $M^{m}$ as a group of isometries such that $\mathbb{R}^{m} / \hat{\Gamma}=M^{m} / G$. Apply the above facts about a finite group action to the present situation. Since the action of $G$ on $M^{m}$ is locally linearized, the stratification $\mathscr{X}$ is locally a product along each stratum; $\dot{a}$ fortiori it satisfies Whitney's conditions. Hence according to Mather [17] (see also [16; pp. 46-50]) we obtain a controlled tube system. Namely, we have the following extra properties and objects.

(4.13) (1) The strata $X$ are locally closed smooth submanifolds defining a locally finite partition of $M^{m}$.

(2) If $X$ meets $\bar{Y}$, then $X \subset \bar{Y}$ (we write $X<Y$ ). (Here $\bar{Y}$ denotes the closure of $Y$.)

(3) Each stratum $X$ has a tubular neighborhood $\left(T_{X}, \pi_{X}, \rho_{X}\right)$; that is, $T_{X}$ is a neighborhood of $X$ in $M^{m}, \pi_{X}: T_{X} \rightarrow X, \rho_{X}: T_{X} \rightarrow[0, \infty]$ are continuous maps, with $X=\rho_{X}^{-1}(0)$.

(4) $\mathrm{T}_{\mathrm{X}}$ meets $T_{Y}$ only if $X<Y, X=Y$ or $Y<X$. Assuming that $X<Y$, $\left(\pi_{X}, \rho_{X}\right)$ defines by restriction a smooth submersion

$$
\left(\pi_{X Y}, \rho_{X Y}\right): T_{X} \cap Y \rightarrow X \times(0, \infty) .
$$

(5) $\pi_{X Y} \circ \pi_{Y Z}=\pi_{X Z}$ and $\rho_{X Y} \circ \pi_{Y Z}=\rho_{X Z}$ (where $X<Y<Z$ ).

In the case at hand, one has more: $\pi_{X}$ and $\rho_{X}$ can be chosen smooth, and $\left(\pi_{X}, \rho_{X}\right): T_{X}-X \rightarrow X \times(0, \infty)$ a smooth submersion. Moreover, it is trivial that the construction can all be done equivariantly.

Furthermore, each tube $T_{X}$ can be identified with an open neighborhood of the 0 -section of a Riemannian vector bundle over $X$ in such a way that $\pi_{X}$ and $\rho_{X}$ become the projection map and quadratic function of the Riemannian metric of the bundle, respectively. 
We say that $X \in \mathscr{X}$ is a proper stratum if $\operatorname{dim} X<\operatorname{dim} M^{m}=m$. Let $X_{1}, X_{2}, \ldots, X_{s}$ be an enumeration of the proper strata in $\mathscr{X}$ such that $X_{i}<X_{j}$ implies that $j<i$, and abbreviate $T_{X_{i}}, \rho_{X_{i}}, \pi_{X_{i}}$ by $T_{i}, \rho_{i}, \pi_{i}$, respectively. If $r \geqslant 0$ define $T_{i}[r]$ and $T_{i}(r)$ by

$$
T_{i}[r]=\left\{x \in T_{i} \mid \rho_{i}(x) \leqslant r\right\} \quad \text { and } \quad T_{i}(r)=\left\{x \in T_{i} \mid \rho_{i}(x)<r\right\} .
$$

Given positive numbers $r_{i+1}, \ldots, r_{s}$, define

$$
T_{i}\left[r ; r_{i+1}, \ldots, r_{s}\right]=T_{i}[r]-\bigcup_{k=i+1}^{s} T_{k}\left(r_{k}\right) ;
$$

and since $T_{i}[0]=X_{i}$, let

$$
X_{i}\left[r_{i+1}, \ldots, r_{s}\right]=T_{i}\left[0 ; r_{i+1}, \ldots, r_{s}\right]
$$

If $0 \leqslant i \leqslant s$, define

$$
M_{i}\left[r_{i+1}, \ldots, r_{s}\right]=M-\bigcup_{k=i+1}^{s} T_{k}\left(r_{k}\right)
$$

Although $\pi_{i}: T_{i}[r] \rightarrow X_{i}$ may not be the entire closed disc bundle of radius $r$, it is easy to construct a sequence of positive numbers $r_{1}, r_{2}, \ldots, r_{s}$ such that for any sequence of numbers $t_{i}, t_{i+1}, \ldots, t_{s}$ with $r_{k} \leqslant t_{k} \leqslant 5(s+1) r_{k}$ (where $i \leqslant k \leqslant s$ ), we have that

$$
\pi_{i}: T_{i}\left[t_{i} ; t_{i+1}, \ldots, t_{s}\right] \rightarrow X_{i}\left[t_{i+1}, \ldots, t_{s}\right]
$$

is the entire closed disc bundle of radius $t_{i}$; furthermore, $X_{i}\left[t_{i+1}, \ldots, t_{s}\right]$ and $M_{i-1}\left[t_{i}, \ldots, t_{s}\right]$ are smooth codimension-0 submanifolds (perhaps with boundaries or corners) of $X_{i}$ and $M^{m}$, respectively.

Define triples of compact submanifolds of $M^{m}$, denoted by $C_{i} \subset \bar{X}_{i} \subset \hat{X}_{i}$ (for $0 \leqslant i \leqslant s)$, by

$$
\begin{aligned}
& C_{0}=M_{0}\left[4 r_{1}, 4 r_{2}, \ldots, 4 r_{s}\right], \\
& \bar{X}_{0}=M_{0}\left[3 r_{1}, 3 r_{2}, \ldots, 3 r_{s}\right], \\
& \hat{X}_{0}=M_{0}\left[2 r_{1}, 2 r_{2}, \ldots, 2 r_{s}\right] ; \text { and for } i>0, \\
& C_{i}=X_{i}\left[(5 i+4) r_{i+1},(5 i+4) r_{i+2}, \ldots,(5 i+4) r_{s}\right], \\
& \bar{X}_{i}=X_{i}\left[(5 i+3) r_{i+1},(5 i+3) r_{i+2}, \ldots,(5 i+3) r_{s}\right], \\
& \hat{X}_{i}=X_{i}\left[(5 i+2) r_{i+1},(5 i+2) r_{i+2}, \ldots,(5 i+2) r_{s}\right] .
\end{aligned}
$$

Let $q: M^{m} \rightarrow M^{m} / G$ be the natural map. Now $M^{m} / G$ has an induced metric from $M^{m}$ : namely, if $a, b \in M / G$, then we define the distance $d_{M / G}(a, b)$ between $a$ and $b$ in $M / G$ to be $d\left(q^{-1}(a), q^{-1}(b)\right)$. Let $N^{n}$ denote $\mathbb{R}^{n} / \Gamma$ and

$$
g: N^{n}=\mathbb{R}^{n} / \Gamma \rightarrow \mathbb{R}^{m} / \hat{\Gamma}=M^{m} / G
$$


be the projection induced from the given admissible fibering apparatus $\mathscr{A}=(\hat{\Gamma}, \phi, f)$. (To avoid obscuring our argument, we shall assume that the manifold $K$ is not present. Its only use is to avoid technical difficulties when $n \leqslant 4$.)

Note that $q\left(C_{i}\right)$ is a codimension- 0 submanifold of the Riemannian manifold $q\left(\bar{X}_{i}\right)$; we shall eventually apply Theorem 4.1 or Corollary 4.2 using the pairs $q\left(C_{i}\right)$, $q\left(\bar{X}_{i}\right)$ in place of $C, X$.

Define triples of compact codimension-0 submanifolds of $M^{m}$, denoted by $M_{i}^{\prime} \subset \hat{M}_{i} \subset M_{i}$ (for $0 \leqslant i \leqslant s$ ), by

$$
\begin{aligned}
M_{i} & =M_{i}\left[(5 i+5) r_{i+1},(5 i+5) r_{i+2}, \ldots,(5 i+5) r_{s}\right], \\
\hat{M}_{i} & =M_{i}\left[(5 i+6) r_{i+1},(5 i+6) r_{i+2}, \ldots,(5 i+6) r_{s}\right], \\
M_{i}^{\prime} & =M_{i}\left[(5 i+7) r_{i+1},(5 i+7) r_{i+2}, \ldots,(5 i+7) r_{s}\right],
\end{aligned}
$$

and let $N_{1}^{\prime} \subset \hat{N}_{i}<N_{i}$ be codimension-0 submanifolds of $N^{n}$ defined by

$$
N_{i}=g^{-1}\left(q\left(M_{i}\right)\right), \quad \hat{N}_{i}=g^{-1}\left(q\left(\hat{M}_{i}\right)\right), \quad N_{i}^{\prime}=g^{-1}\left(q\left(M_{i}^{\prime}\right)\right) .
$$

Also, define triples of codimension-0 submanifolds of $M^{m}$, denoted by $T_{i}^{\prime} \subset \bar{T}_{i} \subset \hat{T}_{i}$, by

$$
\begin{aligned}
T_{i}^{\prime} & =T_{i}\left[(5 i+2) r_{i} ;(5 i+4) r_{i+1},(5 i+4) r_{i+2}, \ldots,(5 i+4) r_{s}\right], \\
\bar{T}_{i} & =T_{i}\left[(5 i+2) r_{i} ;(5 i+3) r_{i+1},(5 i+3) r_{i+2}, \ldots,(5 i+3) r_{s}\right], \\
\hat{T}_{i} & =T_{i}\left[(5 i+3) r_{i} ;(5 i+2) r_{i+1},(5 i+2) r_{i+2}, \ldots,(5 i+2) r_{s}\right] .
\end{aligned}
$$

Notice that

$$
M_{i+1} \subset M_{i}^{\prime} \cup T_{i+1}^{\prime} .
$$

Since $\pi_{i}: \hat{T}_{i} \rightarrow \hat{X}_{i}$ is $G$-equivariant, it induces a map

$$
\Pi_{i}: q\left(\hat{T}_{i}\right)=\hat{T}_{i} / G \rightarrow \hat{X}_{i} / G=q\left(\hat{X}_{i}\right) .
$$

Consider the map $\hat{p}_{i}$ defined (for $1 \leqslant i \leqslant s$ ) as the composite

$$
g^{-1}\left(q\left(\hat{T}_{i}\right)\right) \stackrel{g}{\longrightarrow} q\left(\hat{T}_{i}\right) \stackrel{\Pi_{i}}{\longrightarrow} q\left(\hat{X}_{i}\right) ;
$$

it is a fiber bundle projection. Furthermore, $p_{i}=\hat{p}_{i} \mid g^{-1}\left(q\left(\bar{T}_{i}\right)\right)$ is also a sub-fiber bundle projection onto $q\left(\bar{X}_{i}\right)$; in particular, we obtain the fibers of $p_{i}$ by deleting collar neighborhoods from the boundaries of fibers of $\hat{p}_{i}$. The fundamental groups of the fibers of $p_{i}$ are $\phi^{-1}\left(G_{x}\right)$ where $x \in \bar{X}_{i} \subset X_{i}$ and $\phi: \Gamma \rightarrow \hat{\Gamma}$ is the homomorphism in the fibering apparatus $\mathscr{A}=(\hat{\Gamma}, \phi, f)$ mentioned in the statement of Theorem 2.1. Since $\mathscr{A}$ is admissible and $\mathbb{Z}\left(\phi^{-1}\left(G_{x}\right)\right)$ is right regular, we have by the Bass-HellerSwan Theorem together with Serre's Theorem (cf. [4]) that $\mathrm{Wh}\left(\phi^{-1}\left(G_{x}\right) \times \mathbb{Z}^{k}\right)=0$ for all $k \geqslant 0$. Consequently, each $p_{i}$ satisfies the condition hypothesized for the fiber 
bundle projection $p$ in Corollary 4.2; we shall eventually apply Corollary 4.2 using each of these $p_{i}$. Also define

$$
p_{0}: g^{-1}\left(q\left(\bar{X}_{0}\right)\right) \rightarrow q\left(\bar{X}_{0}\right)=\bar{X}_{0} / G
$$

to be $g g^{-1}\left(q\left(\bar{X}_{0}\right)\right)$. Then, $p_{0}$ is a fiber bundle projection and the fundamental group of the fiber of $p_{0}$ is $\phi^{-1}(1)$. Hence, by the same reasoning as above, $p_{0}$ satisfies the condition hypothesized for $p$ in Theorem 4.1.

Let $\bar{r}=\min \left\{r_{i} \mid 1 \leqslant i \leqslant s\right\}$. Construct inductively a sequence of pairs of positive numbers $\delta_{i}, \varepsilon_{i}$ (for $0 \leqslant i \leqslant s$ ) subject to the constraints that

$$
\begin{aligned}
& \text { (1) } \varepsilon_{s-1} \ll \bar{r} /(5 s+5) \\
& \text { (2) } \varepsilon_{i} \ll \delta_{i+1}<\varepsilon_{i+1} \\
& \text { (3) } \delta_{i}<\bar{\delta}_{i}
\end{aligned}
$$

where $\bar{\delta}_{i}$ is the number $\delta$ posited in Theorem 4.1 (if $i=0$ ) or Corollary 4.2 (if $i>0$ ) when we set $\varepsilon=\varepsilon_{i}, X=q\left(\bar{X}_{i}\right)$ and $C=q\left(C_{i}\right)$. The symbol $a \ll b$ in (4.28) means the ratio $b / a$ is very large. How large depends on the geometry of the chosen controlled tube system (together with the choice of numbers $r_{i}$ ) for the stratification of $M^{m}=\mathbb{R}^{m} / A$ induced by the $G$-action (and on the order of $G$ ). More precisely, the sizes of the ratios in (4.28) depend on the maximum expanding Lipshitz constants for the maps $\pi_{i}, \rho_{i}$ in (4.18); that is, they depend on the maximum of the ratios $\left|d \pi_{i}(v)\right| /|v|$ and $\left|d \rho_{i}(v)\right| /|v|$ as $v$ varies over all the non-zero vectors tangent to $T_{i}\left[(5 s+5) r_{i} ; r_{i+1}, r_{i+2}, \ldots, r_{s}\right]$, where | | denotes the Riemann metric on $M^{m}$. (The ratios of the diameters of curves in $S$ to their images in $S / G$, where $S$ is any $G$-invariant smooth submanifold of $M^{m}$, are dominated above by a number depending only on $|G|$.) The inductive construction can be started by setting $\varepsilon_{s}=1$ and letting $\delta_{s}$ be any number smaller than both $\bar{\delta}_{s}$ and $\varepsilon_{s}$. Clearly, this construction can be continued to produce pairs $\varepsilon_{i}, \delta_{i}$ (for $0 \leqslant i \leqslant s$ ) satisfying (4.28).

Choose the number $\varepsilon$ posited in Theorem 2.1 to be $\delta_{0}$ and let $\left(W ; \partial_{0} W, \partial_{1} W\right)$ be any $(\mathscr{A}, \varepsilon, h$ )-cobordism (as defined in $\S 2$ ) relative to smooth deformation retractions $h_{t}, k_{t}$ where $\partial_{0} W=N^{n}=\mathbb{R}^{n} / \Gamma$. We shall construct inductively product structures over $N_{0}, N_{1}, \ldots, N_{s}=N$. (See (4.22) for the definition of $N_{i}$; note that Theorem 2.1 is proven when this construction is finished.) We start by applying Theorem 4.1 (setting $\left.\varepsilon=\varepsilon_{0}, C=q\left(C_{0}\right), X=q\left(\bar{X}_{0}\right)\right)$ to the $\left(\delta_{0}, h\right)$-cobordism $(W, N)$ where $p=p_{0}: E=g^{-1}\left(q\left(\bar{X}_{0}\right)\right) \rightarrow q\left(\bar{X}_{0}\right)$ and $U=W$. Since $g\left(N_{0}\right) \subset q\left(C_{0}\right)$, we obtain an $\varepsilon_{0}$-product structure over $g\left(N_{0}\right)$ relative to $h_{1}$, and, in particular, an embedding

$$
F_{0}: \mathscr{N}_{0} \times[0,1] \rightarrow W
$$

satisfying (4.4), where $\mathcal{N}_{0}=g^{-1}\left(g\left(N_{0}\right)^{\varepsilon_{0}}\right)$.

Then, we construct inductively embeddings $F_{i}: N_{i} \times[0,1] \rightarrow W$ (for $1 \leqslant i \leqslant s$ ) where $\mathscr{N}_{i}=N_{i-1}^{\prime} \cup g^{-1}\left(q\left(T_{i}^{\prime}\right)\right)$ is a neighborhood of $N_{i}$ in $N$ (cf. (4.24)). These embeddings will satisfy the following properties:

(4.30) (1) $F_{i} \mid \cdot V_{i} \times 0=$ identity;

(2) $h_{1}^{-1}\left(N_{i}\right) \subset$ image $\left(F_{i}\right)$; 
(3) if $x \in N_{i-1}^{\prime}$ and $t \in[0,1]$, then $F_{i}(x, t)=F_{i-1}(x, t)$;

(4) if $x \in g^{-1}\left(q\left(T_{i}^{\prime}\right)\right)$ and $t \in[0,1]$, then $h_{1}\left(F_{i}(x, t)\right) \in g^{-1}\left(q\left(\hat{T}_{i}\right)\right)$ and the diameter in $q\left(\hat{X}_{i}\right)$ of the path $t \mapsto \hat{p}_{i} h_{1} F_{i}(x, t)$ is less than $2 \varepsilon_{i}$.

(Recall formulae (4.26), (4.23) and (4.19).)

Assuming that $F_{1}, F_{2}, \ldots, F_{i-1}$ satisfying (4.30) have been constructed, we shall use Corollary 4.2 to construct $F_{i}$.

(4.31) Let $\bar{W}$ and $\bar{N}$ denote the closures of $W-F_{i-1}\left(N_{i-1}^{\prime} \times[0,1]\right)$ and $N-N_{i-1}^{\prime}$, respectively.

Define $\bar{C}, \bar{X}, E^{\prime}, \bar{E}, \hat{E}$ and $\bar{p}: \bar{E} \rightarrow \bar{X}$ by

$$
\begin{gathered}
\bar{C}=q\left(C_{i}\right), \quad \bar{X}=q\left(\bar{X}_{i}\right), \quad E^{\prime}=g^{-1}\left(q\left(T_{i}^{\prime}\right)\right), \quad \bar{E}=g^{-1}\left(q\left(\bar{T}_{i}\right)\right), \\
\hat{E}=g^{-1}\left(q\left(\hat{T}_{i}\right)\right) \quad \text { and } p=p_{i} \quad \text { (cf. formula 4.26). }
\end{gathered}
$$

(Note that $E^{\prime}=\bar{p}^{-1}(\bar{C})$.) Let $\bar{U}$ be the interior of $h_{1}^{-1}(\hat{E}) \cap \bar{W}$ inside of $\bar{W}$, $\hat{V}=\bar{N} \cap$ interior $\left(\hat{N}_{i-1}\right)$ and $\hat{F}=F_{i-1} \mid \hat{V} \times[0,1]$.

Homotopies $\bar{h}_{t}, \bar{k}_{t}: \bar{U} \times[0,1] \rightarrow \bar{W}$ are obtained by simple taperings of $h_{t}, k_{t}$ (respectively) into $\hat{F}$. To be more specific, for each $x \in \bar{U}$, there is a number $0 \leqslant t_{x} \leqslant 1$ such that $\bar{h}_{t}(x)=h_{t}(x)$ for $t \leqslant t_{x}$ and $\left\{\bar{h}_{t}(x) \mid t>t_{x}\right\}$ is contained in a line of the form $\{\hat{F}(\bar{x}, s) \mid 0 \leqslant s \leqslant 1\}$. Likewise, $\bar{k}_{t}(x)=k_{t}(x)$ for $t \leqslant t_{x}$ and $\left\{\bar{k}_{t}(x) \mid t>t_{x}\right\}$ is contained in a line of the form $\{\hat{F}(\hat{x}, s) \mid 0 \leqslant s \leqslant 1\}$. The number $t_{x}$ varies continuously with $x$ and depends on the distance between $h_{1}(x)$ and $N_{i-1}^{\prime}$; if the distance is large, $t_{x}=1$; if it is small, $t_{x}=0$. We leave as an exercise the details of this tapering construction so that property (4.7) is satisfied where $\bar{V} \subset \bar{V}$ is an appropriately chosen smaller neighborhood of $\partial \bar{N}$ and $\bar{F}=\hat{F} \mid \bar{V}$. (We give a hint: to define $t_{x}$, make use of the function $x \mapsto P_{i}\left(g\left(h_{1}(x)\right)\right)$ where $P_{i}: q\left(T_{i}\right)=T_{i} / G \rightarrow[0, \infty)$ is induced by $\rho_{i}: T_{i} \rightarrow[0, \infty)$.) Additional consequences of this tapering construction are that

$$
d\left(\hat{p}_{i} h_{1}(x), \hat{p}_{i} \bar{h}_{1}(x)\right) \ll \delta_{i}
$$

for $x \in h_{1}^{-1}(\bar{E}) \cap \bar{W}$, and that $(\bar{W}, \bar{N})$ is a $\left(\delta_{i}, h\right)$-cobordism over the closure of $\bar{C}^{2 c_{i}}$ (relative to $\bar{U}, \bar{h}_{t}, \bar{k}_{t}$ ).

We now apply Corollary 4.2 with $\varepsilon, C, X, W, N, F, U, E, h_{t}, k_{t}$ replaced by $\varepsilon_{i}, \bar{C}$, $\bar{X}, \bar{W}, \bar{N}, \bar{F}, \bar{U}, \bar{E}, \bar{h}_{t}, \bar{k}_{t}$, respectively. In this way, we obtain an $\varepsilon_{i}$-product structure $\tilde{F}$ over $\bar{C}$ relative to $\bar{h}_{1}$ extending $\bar{F}$. Glue these two product structures together to define $F_{i}$ satisfying (4.30); namely, let

$$
F_{i}(x, t)= \begin{cases}F_{i-1}(x, t) & \text { if } x \in N_{i-1}^{\prime}, \\ \tilde{F}(x, t) & \text { if } x \in E^{\prime}=g^{-1}\left(q\left(T_{i}^{\prime}\right)\right) .\end{cases}
$$

This completes the proof of Theorem 2.1.

\section{Final remarks}

An arbitrary virtually poly- $\mathbb{Z}$ group $\Gamma$ can contain elements of finite order different from the identity element. In particular, any finite group belongs to this 
class. Hence, in general, Wh $\Gamma$ does not vanish. But, since a lot is known about the $K$-theory of finite groups, one would like to calculate $\mathrm{Wh} \Gamma$ in terms of the $K$-theory of the finite subgroups of $\Gamma$. Unfortunately, $\mathbb{Z} \Gamma$ is no longer a right regular ring when $\Gamma$ contains non-trivial elements of finite order; hence the Nil-groups in the BassHeller-Swan formula can occur in calculating $W h \Gamma$; these are difficult to calculate. For instance, for any ring $R$, if Nil $R \neq 0$, then Nil $R$ is not finitely generated [11]. This is a major difficulty in extending the techniques of this paper to calculate $\mathrm{Wh} \Gamma$ in terms of the $K$-theory of the finite subgroups of $\Gamma$. In some cases this difficulty can be overcome; for example, it can be shown by the techniques of this paper that Wh $((T \oplus T) \rtimes G)=0$ where $(T \oplus T) \rtimes G$ is the 2-dimensional crystallographic group with holonomy group cyclic of order 3 .

On the other hand, since the rational group ring $\mathbb{Q} \Gamma$ is right regular, the following proposed calculation in terms of sheaf homology is probably true.

Conjecture. Let $\Gamma$ be a crystallographic group; then

$$
K_{0}(\mathbb{Q} \Gamma) \simeq H_{0}\left(\mathbb{R}^{n} / \Gamma ; \mathscr{K}_{0}\right)
$$

where $\mathscr{K}_{0}$ denotes the coefficient sheaf on $\mathbb{R}^{n} / \Gamma$ whose stalk over the orbit $x \Gamma$ is $K_{0}\left(\mathbb{Q} F_{x}\right)$ and $F_{x}$ is the isotropy subgroup of $\Gamma$ fixing $x \in \mathbb{R}^{n}$.

\section{References}

1. L. Auslander, et. al., Flows on homogeneous spaces, Annals of Mathematics Studies, 53 (Princeton University Press, Princeton, 1963).

2. L. Auslander, "The structure of solvmanifolds", Bull. Amer. Math. Soc., 79 (1973), 227-261.

3. L. Auslander and F. E. A. Johnson, "On a conjecture of C. T. C. Wall", J. London Math Soc. (2), 14 (1976), 331-332.

4. H. Bass, A. Heller and R. Swan, "The Whitehead group of a polynomial extension", Publications Mathématiques 22 (Institut des Hautes Études Scientifiques, Paris, 1964), pp. 61-79.

5. G. Bredon, Introduction to compact transformation groups (Academic Press, New York, 1972).

6. P. E. Conner and F. Raymond, "Actions of compact Lie groups on aspherical manifolds", Topology of Manifolds (ed. J. C. Cantrell and C. H. Edwards, Jr., Markham, Chicago, 1969), pp. 227-264.

7. P. E. Conner and F. Raymond, "Deforming homotopy equivalences to homeomorphisms in aspherical manifolds", Bull. Amer. Math. Soc., 83 (1977), 36-85.

8. M. Davis, Multiaxial actions on manifolds, Lecture Notes in Mathematics, 643 (Springer, Berlin 1978).

9. D. Epstein and M. Shub, "Expanding endomorphism of flat manifolds", Topology, 7 (1968), 139-141.

10. D. Farkas, "Miscellany on Bieberbach group algebras", Pacific J. Math., 59 (1975), 427-435.

11. F. T. Farrell, "The nonfiniteness of Nil", Proc. Amer. Math. Soc., 65 (1977), 215-216.

12. F. T. Farrell and W. C. Hsiang, "A formula for $K_{1}\left(R_{x}[T]\right)$ ", Applications of categorical algebra, Proceedings of Symposia in Pure Mathematics 17 (American Mathematical Society, Providence, 1970), pp. 192-218.

13. F. T. Farrell and W. C. Hsiang, "Rational L-groups of Bieberbach groups", Comment. Math. Helv., 52 (1977), 89-109.

14. F. T. Farrell and W. C. Hsiang, "The topological-Euclidean space form problem", Invent. Math., 45 (1978), 181-192.

15. F. T. Farrell and W. C. Hsiang, "On the rational homotopy groups of the diffeomorphism groups of discs, spheres and aspherical manifolds", Algebraic and geometric topology, Proceedings of Symposia in Pure Mathematics 32 (American Mathematical Society, Providence, 1978), pp. 325337.

16. C. G. Gibson, K. Wirthmüller, A. A. du Plessis and E. J. N. Looijenga, Topological stability of smooth mappings, Lecture Notes in Mathematics 552 (Springer, Berlin, 1976).

17. J. N. Mather, Notes on topological stability, Lecture Notes, Harvard University, 1970.

18. F. Quinn, "Ends of maps I", Ann. of Math., 110 (1979), 275-331. 
19. F. Raymond and D. Wigner, "Constructions of aspherical manifolds", Geometric applications of homotopy theory I, Lecture Notes in Mathematics 658 (Springer, Berlin, 1978).

20. F. Waldhausen, "Whitehead groups of generalized free products", Algebraic K-theory II, Lecture Notes in Mathematics, 342 (Springer, Berlin, 1973), pp. 155-179.

21. C. T. C. Wall, Surgery on compact manifolds (Academic Press, London, 1971).

Department of Mathematics, The University of Michigan, Ann Arbor, Michigan 48109, U.S.A.
School of Mathematics,

Princeton University and Institute for Advanced Study, Princeton, N.J. 08540, U.S.A. 